\title{
A CRITERION FOR THE ABSOLUTE CONTINUITY OF THE HARMONIC MEASURE ASSOCIATED WITH AN ELLIPTIC OPERATOR
}

\author{
R. FEFFERMAN
}

\section{INTRODUCTION}

In this note, we consider elliptic operators $L$ of the form

$$
L=\sum_{i, j=1}^{n} \partial_{i}\left(a_{i j} \partial_{j}\right)
$$

where the coefficients $a_{i j}$ are defined in some bounded open set $\Omega \subseteq \mathbb{R}^{n}$, are measurable, and satisfy the ellipticity and boundedness condition

$$
\lambda|\xi|^{2} \leq \sum_{i, j=1}^{n} a_{i j}(x) \xi_{i} \xi_{j} \leq \lambda^{-1}|\xi|^{2}
$$

for some $\lambda>0$ and for all $x \in \Omega$ and $\xi \in \mathbb{R}^{n}$. We also assume $a_{i j}=a_{j i}$.

For such operators, the Dirichlet problem is solvable in $\Omega$ if and only if it is solvable for the Laplace operator, according to a theorem of Littman, Stampacchia and Weinberger [1]. This means that if $\Omega \subseteq \mathbb{R}^{n}$ is a sufficiently nice bounded region (the unit ball, $B$, is an example) and $f$ is a given continuous function on the boundary of $\Omega$, then there exists a unique function $u$, continuous on $\bar{\Omega}$, so that $L(u)=0$ in $\Omega$ and $u=f$ on $\partial \Omega$. Let us assume, for convenience, that the origin belongs to $\Omega$. Then the mapping $f \in \mathscr{C}(\partial \Omega) \rightarrow u(0)$ is a positive linear functional so there exists a unique nonnegative measure $\omega$ on $\partial \Omega$ such that for every $f \in \mathscr{C}(\partial \Omega)$,

$$
\int_{\partial \Omega} f d \omega=u(0) \text {. }
$$

This measure $\omega$ is called the harmonic measure associated to $L$. It is often important for applications to know whether or not $\omega$ is absolutely continuous with respect to the surface measure $d \sigma$ on the boundary of $\Omega$. If this is the case, it is also of interest to know how nice the Radon-Nikodym derivative $d \omega / d \sigma$ (the Poisson kernel) is.

In recent years, several results have been found to answer these questions. First, according to a result of Caffarelli, Fabes, and Kenig [2] there exist elliptic

Received by the editors May 22, 1988.

1980 Mathematics Subject Classification. Primary 35J25.

(C)1989 American Mathematical Society 
operators $L$ of the form (1) such that the measure $\omega$ associated to $L$ is not absolutely continuous with respect to $\sigma$. Later, Fabes, Jerison, and Kenig [3] proved the following theorem:

Suppose $L=\sum_{i, j=1}^{n} \partial_{i}\left(a_{i j} \partial_{j}\right)$, where the $a_{i j}$ satisfy (2). Suppose further that the coefficient matrices $A(x)=\left(a_{i j}(x)\right)$ are continuous on $\bar{B}$. Let the modulus of continuity $\eta$ be defined for $0<t<1$ by

$$
\eta(t)=\sup _{\substack{x \in \partial B \\ 0<s \leq t}}\|A(x-s x)-A(x)\| .
$$

Assume that $\int_{0}^{1} \eta^{2}(t) d t / t<\infty$. Then $\omega$ is absolutely continuous with respect to $\sigma$. In addition, if $d \omega=k d \sigma$ then $k$ satisfies the reverse Hölder inequality

$$
\left(\frac{1}{\sigma(\Delta)} \int_{\Delta} k^{2} d \sigma\right)^{1 / 2} \leq C \frac{1}{\sigma(\Delta)} \int_{\Delta} k d \sigma
$$

for all surface balls $\Delta \subseteq \partial B$.

Then in [4], Dahlberg extended this result to the case where the coefficients of $L$ are discontinuous, and to a more general setting. To describe his result, let us recall several definitions. If $\Delta \subseteq \partial B$ is a surface ball centered at $x \in \partial B$, of radius $r$, then we set $S(\Delta)=B(x ; r) \cap B$. A nonnegative measure $\mu$ in $B$ is called a Carleson measure if and only if $\mu(S(\Delta)) \leq C \sigma(\Delta)$ for all surface balls $\Delta \subseteq \partial B$. We say that $\mu$ is a Carleson measure with vanishing trace provided there exists a function $h(r)$ for $0<r<1$, such that $\lim _{r \rightarrow 0} h(r)=0$ and $\mu(S(\Delta)) \leq h(r) \sigma(\Delta)$ for all surface balls $\Delta$ of radius $r$, and all $0<r<1$. If $f(x)$ is a nonnegative function on $\partial B$, then we say that $f \in B^{p}$ for some $1<p<\infty$ if and only if for all surface balls $\Delta$,

$$
\left(\frac{1}{\sigma(\Delta)} \int_{\Delta} f^{p} d \sigma\right)^{1 / p} \leq C\left(\frac{1}{\sigma(\Delta)} \int_{\Delta} f d \sigma\right) .
$$

The smallest $C$ for which (4) is valid is called the " $B^{p}$ norm" of $f$.

In [4], Dahlberg proves the following theorem:

Suppose $L_{0}$ and $L_{1}$ are two operators of the form (1), with coefficient matrices $A_{0}(x)$ and $A_{1}(x)$ respectively. For $z \in B$ set

$$
a(z)=\sup _{x \in B(z ; \delta(z) / 2)}\left\|A_{0}(x)-A_{1}(x)\right\|,
$$

where $\delta(z)$ denotes the distance from $z$ to $\partial B$. Assume that $a^{2} d z / \delta$ is a Carleson measure with vanishing trace. Assume that the harmonic measure $\omega_{0}$ associated with $L_{0}$ is absolutely continuous with respect to $\sigma$ and that $d \omega_{0}=$ $k_{0} d \sigma$ where $k_{0} \in B^{p}, 1<p<\infty$. Then the same must be true of the harmonic measure $\omega_{1}$ associated to $L_{1}$, i.e., $\omega_{1}=k_{1} d \sigma$ for some $k_{1} \in B^{p}$.

To state our result, let us recall that a function $f(x) \geq 0$ on $\partial B$ belongs to $A^{\infty}$ if and only if for $d \nu=f d \sigma$, and any subset $E \subseteq \Delta, \Delta$ a surface ball, we 
have

$$
\frac{\nu(E)}{\nu(\Delta)} \leq C\left[\frac{\sigma(E)}{\sigma(\Delta)}\right]^{\theta}, \quad \text { for some } \theta>0 .
$$

Then it is well known, [5], that $f \in A^{\infty}$ if and only if $f \in B^{p}$ for some $p>1$.

The result of this article will give a criterion which, on the formal level, looks very much like the Dini condition of Fabes, Jerison, and Kenig which guarantees that if the difference of the coefficient matrices $A_{0}$ and $A_{1}$ of two elliptic operators $L_{0}$ and $L_{1}$ meets the criterion, then $\omega_{0}=k_{0} d \sigma, k_{0} \in A^{\infty}$ implies that $\omega_{1}=k_{1} d \sigma$, with $k_{1} \in A^{\infty}$. We are not able to prove that the $B^{p}$ condition is preserved for a given $p$. Thus the conclusion of the theorem of Dahlberg is stronger than ours here. The significance of our result comes from the fact that, unlike the results in [3] and [4] our hypothesis does not require that the coefficients of $L_{0}$ be uniformly close to those of $L_{1}$ as we approach the boundary of $B$, in order to guarantee that the good properties of the harmonic measure associated with $L_{0}$ are inherited by that of $L_{1}$.

\section{Statement of Theorem}

Suppose that $L_{0}$ and $L_{1}$ are elliptic operators in divergence form (1) with bounded measureable coefficients defined in the unit ball, $B$. Suppose, as above, $A_{0}(x)$ and $A_{1}(x)$ denote their coefficient matrices, and $\omega_{0}$ and $\omega_{1}$ the associated harmonic measures.

As above, for $z \in B$ set

$$
a(z)=\sup _{x \in B(z ; \delta(z) / 2)}\left\|A_{0}(x)-A_{1}(x)\right\|
$$

where $\delta(z)$ is the distance of $z$ to the boundary of $B$. Then we have the following:

Theorem. Suppose, for each $x \in \partial B$,

$$
\int_{0}^{1} a^{2}((1-t) x) \frac{d t}{t} \leq C,
$$

for some constant $C$ independent of $x$. Assume that $\omega_{0}=k_{0} d \sigma$ where $k_{0} \in$ $A^{\infty}$. Then $\omega_{1}$ is absolutely continuous with respect to $\sigma$ and $\omega_{1}=k_{1} d \sigma$ where $k_{1} \in A^{\infty}$.

The proof of our theorem follows the method of Dahlberg in [4]. There, Dahlberg considers the family of operators $L_{t}, 0 \leq t \leq 1$, given by $L_{t}=$ $(1-t) L_{0}+t L_{1}$. Let $\omega_{t}$ denote the harmonic measure associated to $L_{t}$ and $Q(t)$ the $B^{p}$ norm of $\omega_{t}$. He proves the differential inequality $|\dot{Q}(t)| \leq C Q(t)^{N}$ where $\dot{Q}(t)$ is the $t$ derivative of $Q(t), C$ depends on the Carleson measure constant of $a^{2} d z / \delta$, and $\lambda$, the ellipticity constant. $N$ is some large positive integer. This differential inequality shows that if $a^{2} d z / \delta$ is a Carleson measure of vanishing trace (so that essentially $C$ can be taken as small as desired) and 
$Q(0)<\infty$, then $Q(1)<\infty$. The smallness of $C$ is obviously crucial, since the solution of the equation $|\dot{Q}(t)|=C Q(t)^{N}$ has a singularity when $N>1$. Our aim here is to replace the differential inequality above with $|\dot{Q}(t)| \leq C Q(t)$, i.e., $N=1$. $C$ can then be as large as we like.

As one application of our theorem, we introduce the following notion, which we call "region of arbitrary perturbation." Let $\Omega \subseteq \mathbb{R}^{n}$ be a bounded region in which the classical Dirichlet problem is solvable, and let $\Omega_{0} \subseteq \Omega$ be a subregion. We call $\Omega_{0}$ a region of arbitrary perturbation provided whenever $L_{0}$ and $L_{1}$ are two elliptic operators of the form (1) with coefficients $A_{0}(x)$ and $A_{1}(x)$, then if $A_{0}(x)-A_{1}(x)$ is supported in $\Omega_{0}$, and if the harmonic measure associated with $L_{0}$ is $A^{\infty}$ on $\partial \Omega$, then this implies that the same is true for the harmonic measure associated with $L_{1}$. Now set $B_{+} \subseteq \mathbb{R}^{n+1}$ to be

$$
B_{+}=\left\{(x, y) \mid x \in \mathbb{R}^{n}, y>0, \text { and }|x|^{2}+y^{2}<1\right\}
$$

and

$$
B_{+}\left(\frac{1}{2}\right)=\left\{(x, y) \mid x \in \mathbb{R}^{n}, y>0 \text { and }|x|^{2}+y^{2}<\frac{1}{2}\right\} .
$$

Suppose $\varphi(x)$ is a function defined on the ball centered at 0 of radius $\frac{1}{4}$ in $\mathbb{R}^{n}$ whose graph is contained in $B_{+}\left(\frac{1}{2}\right)$ and which is slowly oscillating in the sense that there exists a constant $C$ so that for each $x_{0} \in B_{1 / 4}(0)$, we have $\frac{1}{2} \varphi\left(x_{0}\right) \leq \varphi(x) \leq 2 \varphi\left(x_{0}\right)$ whenever $x \in B_{\left|x_{0}\right| / C}\left(x_{0}\right) \cap B_{1 / 4}(0)$. Let

$$
\Omega_{0}=\left\{\left.(x, y)|| x\right|^{2}+y^{2}<\frac{1}{4} \text { and } \varphi(x)<y<2 \varphi(x)\right\} .
$$

Then $\Omega_{0}$ is a region of arbitrary perturbation, as can easily be seen from our theorem.

The notion of regions of arbitrary perturbation can be used to yield information on the harmonic measure associated with some basic examples of elliptic operators. A discussion of these will appear elsewhere.

Proof of the Theorem. Consider $L_{0}$ and $L_{1}$ as in the statement of our theorem, and let $L_{t}=(1-t) L_{0}+t L_{1}$ with associated harmonic measure $\omega_{t}$. In [4] it is shown that we may assume that the coefficients of the $L_{t}$ are $C^{\infty}$ in $B$. Let $\omega_{t}=k_{t} d \sigma$, and $\Delta$ be a surface ball on $\partial B, \Delta$ centered at $x_{0}$ of radius $r$. Let $A=B\left(x_{0} ; 2 r\right) \cap B$. We are trying to show that if $k_{0} \in A^{\infty}$ then the same is true of $k_{1}$. To this end, let $\tilde{\sigma}$ be the normalized surface measure on $\Delta$, i.e., $\tilde{\sigma}=\sigma / \sigma(\Delta)$. We shall show that

$$
\left\|k_{1}\right\|_{L(\log L)(d \grave{\sigma})} \leq C\left\|k_{1}\right\|_{L^{\prime}(d \tilde{\sigma})}
$$

and this shows that $k_{1} \in A^{\infty}$. To do this, we require a trivial lemma, which the reader will notice is essentially just exploiting the duality of $H^{1}$ with BMO.

Lemma. Fix $t_{0} \in[0,1]$, and $\Delta \subseteq \partial B$ a surface ball centered at $x_{0}$ of radius $r$. There exists a function $f(x)$ defined on the boundary of $B$, which is continuous, 
nonnegative, supported in the surface ball centered at $x_{0}$ of radius $\frac{3}{2} r, \widetilde{\Delta}$, such that

$$
\|f\|_{\mathrm{BMO}(d \sigma)}+\|f\|_{L^{\prime}(d \sigma / \sigma(\Delta))} \leq C_{n}
$$

and

$$
\int_{\tilde{\Delta}} f k_{t_{0}} d \tilde{\sigma} \geq c_{n}\left\|k_{t_{0}}\right\|_{L(\log L)(d \grave{\sigma})} .
$$

(Here $C_{n}, c_{n}$ depend only on $n$.)

Proof of Lemma. We clearly may assume, by homogeneity, that $\left\|k_{t_{0}}\right\|_{L^{\prime}(d \grave{\sigma})}=1$. By a well-known theorem of E. M. Stein [6],

$$
\left\|k_{t_{0}}^{*}\right\|_{\left.L^{\prime} d \tilde{\sigma}\right)} \geq A_{n}\left\|k_{t_{0}}\right\|_{L(\log L)(d \tilde{\sigma})}
$$

where $k_{t_{0}}^{*}$ denotes the Hardy-Littlewood (dyadic) maximal function of $k_{t_{0}}$ taken over cubes contained in $\Delta$. Perform the Calderòn-Zygmund decomposition of the $k_{t_{0}}$ at heights $B^{j}, j=1,2, \ldots$, where $B$ is as follows:

Let the Calderon-Zygmund cubes at height $B^{j}$ be called $Q_{\ell}^{j}$. Then choose $B$ so large that

$$
\sigma\left(Q_{\ell}^{j} \cap\left[\bigcup_{m} Q_{m}^{j+1}\right]\right)<\frac{1}{2} \sigma\left(Q_{\ell}^{j}\right) .
$$

Then let $\varphi_{\ell}^{j}$ be a nonnegative smooth bump function which is 1 on $Q_{\ell}^{j}$ and 0 off of the dilate of $Q_{\ell}^{j}$ by $3 / 2$. It is trivial to show that the function $f=$ $\sum_{j . \ell} \varphi_{\ell}^{j}+\varphi$ belongs to $\mathrm{BMO}(d \sigma)\left(\varphi \in C^{\infty}(\partial B)\right), \varphi \geq 0, \varphi \equiv 1$ on $\Delta$, and is supported in the concentric dilate of $\Delta$ by $3 / 2$ ) and

$$
\|f\|_{\mathrm{BMO}(d \sigma)}+\|f\|_{L^{\prime}(d \grave{\sigma})} \leq C_{n},
$$

while

$$
\begin{aligned}
\int f k_{t_{0}} d \sigma & \geq \int_{\Delta} k_{t_{0}} d \tilde{\sigma}+\sum_{j, \ell} \int_{Q_{1}^{\prime}} k_{t_{0}} d \tilde{\sigma} \\
& \geq \frac{1}{\sigma(\Delta)}\left[\sigma(\Delta)\left(\frac{1}{\sigma(\Delta)} \int_{\Delta} k_{t_{0}} d \sigma\right)+\sum_{j, \ell} \sigma\left(Q_{\ell}^{j}\right)\left(\frac{1}{\sigma\left(Q_{\ell}^{j}\right)} \int_{Q^{\prime}} k_{t_{0}} d \sigma\right)\right] \\
& \geq \frac{1}{\sigma(\Delta)} c_{n}^{\prime}\left[\sigma(\Delta)\left(\frac{1}{\sigma(\Delta)} \int_{\Delta} k_{t_{0}} d \sigma\right)+\sum_{j, \ell} B^{j} \sigma\left(Q_{\ell}^{j}\right)\right] \\
& \geq c_{n}^{\prime \prime} \int_{\Delta} k_{t_{0}}^{*} d \tilde{\sigma} \geq c_{n}^{\prime \prime} A_{n}\left\|k_{t_{0}}\right\|_{L \log L(d \tilde{\sigma})} .
\end{aligned}
$$

This proves the lemma.

Now, to show (5), we fix $t_{0} \in[0,1]$ and $\Delta$, and we select $f$ as in the lemma, and estimate

$$
\int_{\tilde{\Delta}} f k_{t_{0}} d \sigma / \int_{\Delta} k_{t_{0}} d \sigma
$$


and this ratio is shown in Dahlberg [4] to be equivalent to a quantity $V$ as follows: Let $u_{t}$ be the solution of $L_{t} u_{t}=0$ in $A$ and $u_{t}=f$ on $\partial A$. Let $\psi(z) \in$ $C^{\infty}\left(\mathbb{R}^{n}\right), \psi$ supported inside the ball $B\left(0 ; \frac{1}{2} r\right), \psi \equiv 2$ inside $B\left(0 ; \frac{1}{4} r\right)$. Let $\bar{z}=\left(1-\frac{3}{2} r\right) x_{0}$ and $\psi(z)=\psi((s-\bar{z}) / r) r^{-n}$. Put $V=\int_{A} u_{t_{0}} \psi d z$. We have $c_{1} V \leq(6) \leq c_{2} V$, so we estimate (6) by estimating $V$. Following [4] we control $\int_{A} u_{t} \psi d z$, via a differential inequality. We consider the modified Green's function $h_{t}$ defined by $L_{t} h_{t}=\psi$ in $A$ and $h_{t} \equiv 0$ on $\partial A$. Then as in [4],

$$
\left|\int_{A} u_{t} \psi d z\right| \leq C \int_{A} \varepsilon\left|\nabla u_{t} \| \nabla h_{t}\right| d z
$$

where $\varepsilon(z)=\left\|A_{0}(z)-A_{1}(z)\right\|$.

Now, our assumption on $a$ implies that $\|\varepsilon\|_{L^{\infty}} \leq C$. Let $A_{1}=B\left(x_{0} ; \frac{3}{2} r\right) \cap B$ and $A_{2}=A-A_{1}$. Then

$$
\int_{A_{2}} \varepsilon\left|\nabla u_{t}\right|\left|\nabla h_{t}\right| d z \leq C\left(\int_{A_{2}}\left|\nabla u_{t}\right|^{2} d z\right)^{1 / 2}\left(\int_{A_{2}}\left|\nabla h_{t}\right|^{2} d z\right)^{1 / 2}
$$

and this is easily seen to be

$$
\leq C \int_{\widetilde{\Delta}} f k_{t} d \sigma
$$

where in this case and from here on $\omega_{t}$ will stand for the harmonic measure associated with $L_{t}$ in $A$ taken at the point $\bar{z}$, and $\omega_{t}=k_{t} d \sigma$. Then

$$
\begin{aligned}
\int_{\widetilde{\Delta}} f k_{t} d \sigma & \leq C \sigma(\Delta) \int_{\widetilde{\Delta}} f k_{t} \frac{d \sigma}{\sigma(\widetilde{\Delta})} \\
& \leq C \sigma(\Delta) \int_{\widetilde{\Delta}}\left|f-f_{\widetilde{\Delta}}\right| k_{t} \frac{d \sigma}{\sigma(\widetilde{\Delta})}+C \sigma(\Delta) f_{\widetilde{\Delta}} \int_{\widetilde{\Delta}} k_{t} \frac{d \sigma}{\sigma(\widetilde{\Delta})}
\end{aligned}
$$

(where $\left.f_{\widetilde{\Delta}}=\int_{\widetilde{\Delta}} f d \sigma / \sigma(\widetilde{\Delta})\right)$

$$
\begin{aligned}
& \leq C \sigma(\Delta)\left\|f-f_{\widetilde{\Delta}}\right\|_{\exp (d \tilde{\sigma})}\left\|k_{t}\right\|_{L \log L(d \tilde{\sigma})}+C \\
& \leq C\|f\|_{\mathrm{BMO}(d \sigma)} Q(t)\left\|k_{t}\right\|_{L^{1}(d \sigma / \sigma(\Delta))} \sigma(\Delta)+C \leq C^{\prime} Q(t)
\end{aligned}
$$

where

$$
Q(t)=\sup _{\Delta \subseteq \partial B} \frac{\left\|k_{t}\right\|_{L \log L(d \sigma / \sigma(\Delta) ; \Delta)}}{\left\|k_{t}\right\|_{L^{\prime}(d \sigma / \sigma(\Delta) ; \Delta)}}
$$

the sup being taken over all surface balls $\Delta \subseteq \partial B$.

Now we must estimate $\int_{A_{1}} \varepsilon\left|\nabla u_{t}\right|\left|\nabla h_{t}\right| d z$. For each dyadic surface cube $Q \subseteq \widetilde{\Delta}$, we let

$$
\begin{array}{r}
\widehat{Q}=\left\{z \in B \mid z /\|z\| \in Q, c_{n} \ell \leq \delta(z)<2 c_{n} \ell, \ell=\text { side length of } Q\right\}, \\
c_{n}=10 \sqrt{n} .
\end{array}
$$


Then if $a_{Q}=\sup \{\varepsilon(z) \mid z \in \widehat{Q}\}$,

$$
\begin{aligned}
\int_{A_{1}} \varepsilon\left|\nabla u_{t}\right|\left|\nabla h_{t}\right| d z & \leq \sum_{\substack{Q \subseteq \widetilde{\Delta} \\
Q \text { dyadic }}} \int_{\widehat{Q}} \varepsilon\left|\nabla u_{t}\right|\left|\nabla h_{t}\right| d z \\
& \leq \sum_{\substack{Q \subseteq \widetilde{\Delta} \\
Q \text { dyadic }}} a_{Q}\left(\int_{\widehat{Q}}\left|\nabla u_{t}\right|^{2} d z\right)^{1 / 2}\left(\int_{\widehat{Q}}\left|\nabla h_{t}\right|^{2} d z\right)^{1 / 2} .
\end{aligned}
$$

By Caccioppoli's inequality,

$$
\left(\int_{\widehat{Q}}\left|\nabla h_{t}\right|^{2} d z\right)^{1 / 2} \leq C\left(\int_{\widehat{Q}^{\sim}} h_{t}^{2} d z\right)^{1 / 2} \delta(Q)^{-1}
$$

where $\widehat{Q}^{\sim}$ denotes the concentric dilate of $\widehat{Q}$ by $\left(1+\frac{1}{100}\right)$, and $\delta(Q)$ denotes the side length of $Q$.

By standard estimates on Green's function [7], if $z \in \widehat{Q}^{\sim}, h_{t}(z) \leq$ $C \delta(Q)^{2-n} \omega_{t}(Q)$, so that

$$
\left(\int_{\widehat{Q}^{\sim}} h_{t}^{2} d z\right)^{1 / 2} \leq C \delta(Q)^{2-n / 2} \omega_{t}(Q)
$$

so that

$$
\begin{aligned}
\int_{A_{1}} \varepsilon\left|\nabla u_{t}\right|\left|\nabla h_{t}\right| d z & \leq \sum_{\substack{Q \subseteq \widetilde{\Delta} \\
Q \text { dyadic }}} a_{Q}\left(\int_{\widehat{Q}}\left|\nabla u_{t}\right|^{2} d z\right)^{1 / 2} \delta^{1-n / 2}(Q) \omega_{t}(Q) \\
& \leq \sum_{\substack{Q \subseteq \widetilde{\Delta} \\
Q \text { dyadic }}} a_{Q}\left(\int_{\widehat{Q}}\left|\nabla u_{t}\right|^{2} \delta^{2-n}(z) d z\right)^{1 / 2} \omega_{t}(Q)
\end{aligned}
$$

Define $F(x) \in \ell^{2}$ for $x \in \partial B$, by letting $F(x)=\left\{a_{Q}\right\}_{\substack{x \in Q \\ Q \text { dyadic }}}$. Define $G(x) \in \ell^{2}$ for $x \in \partial B$ by

$$
G(x)=\left\{\left(\int_{\widehat{Q}}\left|\nabla u_{t}\right|^{2} \delta^{2-n}(z) d z\right)^{1 / 2}\right\}_{\substack{x \in Q \\ Q \text { dyadic }}} .
$$

Then $(8) \leq \int_{\partial B} F \cdot G(x) d \omega_{t}(x)$. Also, our assumption on $a$ implies that $F \in$ $L^{\infty}\left(\ell^{2}\right)$ and $|G(x)|_{\ell^{2}}=S(f)(x)$.

We therefore see that

$$
(8) \leq C \int_{\partial B} S(f)(x) d \omega_{t}(x) \leq C\left[\int_{\partial B} S^{2}(f)(x) d \omega_{t}(x)\right]^{1 / 2} .
$$

By a result of Dahlberg, Jerison, and Kenig [8], $S$ is bounded on $L^{2}\left(d \omega_{t}\right)$ with a bound depending only on $n$ and the ellipticity constant of $L_{t}$. Thus this last 
quantity is bounded by

(9)

$$
C^{\prime}\left(\int_{\partial B} f^{2} d \omega_{t}\right)^{1 / 2}=C^{\prime}\left(\int_{\widetilde{\Delta}} f^{2} d \omega_{t}\right)^{1 / 2} \leq C^{\prime}\left(\int_{\widetilde{\Delta}}\left|f-\bar{f}_{\widetilde{\Delta}}\right|^{2} d \omega_{t}\right)^{1 / 2}+C^{\prime}\left|\bar{f}_{\widetilde{\Delta}}\right|
$$

where

$$
\bar{f}_{\widetilde{\Delta}}=\frac{1}{\omega_{t}(\widetilde{\Delta})} \int_{\widetilde{\Delta}} f d \omega_{t} .
$$

At this point, we observe that $\omega_{t}$ satisfies a doubling condition on surface balls [7], with doubling constants depending only on the ellipticity constants of $L$, and so

$$
\begin{aligned}
\left(\frac{1}{\omega_{t}(\widetilde{\Delta})} \int_{\widetilde{\Delta}}\left|f-\bar{f}_{\widetilde{\Delta}}\right|^{2} d \omega_{t}\right)^{1 / 2} & \leq C^{\prime \prime} \sup _{\Delta \subseteq \partial B}\left(\frac{1}{\omega_{t}(\Delta)} \int_{\Delta}\left|f-\bar{f}_{\Delta}\right| d \omega_{t}\right) \\
& \equiv C^{\prime \prime}\|f\|_{\mathrm{BMO}\left(d \omega_{t}\right)}
\end{aligned}
$$

where $C^{\prime \prime}$ depends only on $n, C$, and the ellipticity constant of $L_{0}$ and $L_{1}$, and where $\bar{f}_{\Delta}=\left(1 / \omega_{t}(\Delta)\right) \int_{\Delta} f d \omega_{t}$.

Next, we claim that $\|f\|_{\mathrm{BMO}\left(d \omega_{t}\right)} \leq C^{\prime \prime \prime} Q(t)$. In fact, if $Q$ is a surface cube on $\partial B$, and if $f_{Q}=(1 / \sigma(Q)) \int_{Q} f d \sigma$, then

$$
\begin{aligned}
\frac{1}{\omega_{t}(Q)} \int_{Q}\left|f-f_{Q}\right| d \omega_{t} & =\frac{\sigma(Q)}{\omega_{t}(Q)} \int_{Q}\left|f-f_{Q}\right| k_{t} \frac{d \sigma}{\sigma(Q)} \\
& \leq \frac{\sigma(Q)}{\omega_{t}(Q)}\left\|f-f_{Q}\right\|_{\exp (d \sigma / \sigma(Q) ; Q)}\left\|k_{t}\right\|_{L \log L(d \sigma / \sigma(Q) ; Q)} \\
& \leq C \frac{\sigma(Q)}{\omega_{t}(Q)}\|f\|_{\mathrm{BMO}(d \sigma)} Q(t)\left\|k_{t}\right\|_{L^{\prime}(d \sigma / \sigma(Q) ; Q)} \\
& \leq C^{\prime \prime \prime} Q(t)
\end{aligned}
$$

proving the claim.

Finally

$$
(9) \leq \bar{C} Q(t)+C^{\prime} \frac{1}{\omega_{t}(\widetilde{\Delta})} \int_{\widetilde{\Delta}} f k_{t} d \sigma \leq \bar{C} Q(t)+C^{\prime} \frac{\sigma(\widetilde{\Delta})}{\omega_{t}(\widetilde{\Delta})} \int_{\widetilde{\Delta}} f k_{t} \frac{d \sigma}{\sigma(\widetilde{\Delta})} .
$$

But

$$
\int_{\widetilde{\Delta}} f k_{t} \frac{d \sigma}{\sigma(\widetilde{\Delta})} \leq \int_{\widetilde{\Delta}}\left|f-f_{\widetilde{\Delta}}\right| k_{t} \frac{d \sigma}{\sigma(\widetilde{\Delta})}+f_{\widetilde{\Delta}} \int_{\widetilde{\Delta}} k_{t} d \sigma
$$

(where $\left.f_{\widetilde{\Delta}}=(1 / \sigma(\widetilde{\Delta})) \int_{\widetilde{\Delta}} f d \sigma \leq C\right)$

$$
\begin{aligned}
& \leq\|f\|_{\mathrm{BMO}(d \sigma)}\left\|k_{t}\right\|_{L \log L(d \sigma / \sigma(\widetilde{\Delta}) ; \widetilde{\Delta})}+C \\
& \leq C Q(t)\left\|k_{t}\right\|_{L^{\prime}(d \sigma / \sigma(\widetilde{\Delta}) ; \widetilde{\Delta})}+C .
\end{aligned}
$$

From this it follows that $(10) \leq C Q(t)$. This proves that $\left|\int_{A} u_{t} \psi d z\right| \leq$ $C Q(t)$. 
Now we have shown that

$$
\int_{A} u_{t_{0}} \psi d z \leq \int_{A} u_{0} \psi d z+\int_{0}^{t_{0}}\left(\int_{A} u_{t} \psi d z\right) d t \leq C\left[Q(0)+\int_{0}^{t_{0}} Q(t) d t\right] \text {, }
$$

while $Q\left(t_{0}\right) \leq C \int_{A} u_{t_{0}} \psi d z$, if we choose $\Delta$ correctly, so that

$$
Q\left(t_{0}\right) \leq C\left[Q(0)+\int_{0}^{t_{0}} Q(t) d t\right]
$$

and this implies the bound on $Q(1)$ we require to finish the proof of the theorem.

\section{REFERENCES}

1. W. Littman, G. Stampacchia, and H. Weinberger, Regular points for elliptic equations with discontinuous coefficients, Ann. Scuola Norm. Sup. Pisa 17 (1963), 45-79.

2. L. Caffarelli, E. Fabes, and C. Kenig, Completely singular elliptic harmonic measures, Indiana Univ. Math. J. 30 (1981), 917-924.

3. E. Fabes, D. Jerison, and C. Kenig, Necessary and sufficient conditions for absolute continuity of elliptic-harmonic measure, Ann. of Math. 119 (1984), 121-141.

4. B. Dahlberg, On the absolute continuity of elliptic measures, Amer. J. Math. 108 (5) (1986), $1119-1138$.

5. R. Coifman and C. Fefferman, Weighted norm inequalities for maximal functions and singular integrals, Studia Math. 51 (1974), 241-250.

6. E. M. Stein, Note on the class $L \log L$, Studia Math. 31 (1969), 305-310.

7. L. Caffarelli, E. Fabes, S. Mortella, and S. Salsa, Boundary behavior of non-negative solutions of elliptic operators in divergence form, Indiana Univ. Math. J. 30 (1981), 621-640.

8. B. Dahlberg, D. Jerison, and C. Kenig, Area integral estimates for elliptic differential operators with non-smooth coefficients, Arkiv fur Matematik 22 (1984), 97-108.

9. F. John and L. Nirenberg, On functions of bounded mean oscillation, Comm. Pure Appl. Math. 14 (1961), 415-426.

Department of Mathematics, University of Chicago, 5734 S. University Avenue, Chicago, Illinois 60637 